\title{
Introducción: La didáctica de las matemáticas en los 90
}

\section{Carmen Gómez}

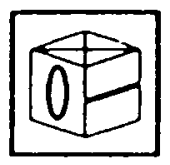

La aritmética no era mi fuerte, me parecía que los problemas no tenian ningún sentido: iqué cantidad de frutas se obtiene cuando llenamos un cesto con tres cuartos de manzanas, un octavo de melocotones y dos sextos de otra cosa? Yo no veía el problema; me preguntaba por qué debian baber llenado un cesto de aquella forma; no tenia, pues, solución.

Marguerite Yourcenar

No parece muy arriesgado afirmar que si hiciéramos una encuesta entre la población, un alto porcentaje de personas suscribiría con gusto la frase de Yourcenar. «¿Por qué debían haber llenado el cesto de aquella manera?» Quizá en esta pregunta tan simple reside la clave para responder a otra pregunta: ¿Por qué para la mayoría de la gente las matemáticas son difíciles, aburridas e incomprensibles? Pensar «matemáticamente» exige sin duda pensar de forma muy diferente a como lo hacemos en la vida cotidiana.

Si esta afirmación es válida para todo conocimiento científico, lo es mucho más para el conocimiento matemático, por su alto nivel de abstracción y desvinculación de lo real. ¿Es posible conseguir que el acercamiento al razonamiento formal matemático se realice de forma menos «traumática»?

Aunque en los últimos años la psicología del pensamiento ha aportado abundante información sobre las ideas propias, preconceptos, conocimientos no formales, prerrequisitos, etc., que poseen los niños acerca de los diferentes aspectos de la realidad, sabemos muy poco sobre las formas en que las personas aprenden, tanto en contextos de instrucción formal como no formal.

En este número que presentamos no pretendemos, obviamente, dar respuesta a tan complejas cuestiones, pero sí abrir un debate y aportar elementos a una discusión - la de la enseñanza de las matemáticas - que nos parece hoy más que nunca urgente y relevante por dos razones distintas.

La primera porque, como se señala en alguno de los artículos de este volumen, estamos en presencia de una curiosa paradoja: por una parte nuestras sociedades 


\section{8}

occidentales, cada vez más desarrolladas científica y tecnológicamente, exigen un nivel alto de conocimiento matemático y valoran éste como uno de los conocimientos más importantes, por no decir el que más; por otra, cada día es mayor la conciencia, avalada por numerosos estudios y estadísticas, de que el conocimiento matemático de la población es ínfimo y que el «analfabetismo matemático» nos invade.

La segunda, porque en un momento de Reforma Educativa que plantea cambios curriculares importantes, tanto en los contenidos como en la metodología de enseñanza de las matemáticas, es conveniente abrir un debate sobre el significado y la viabilidad de dichos cambios.

Con este objetivo de promover, ampliar y colaborar al debate, los artículos recogidos en este volumen responden a una amplia gama de enfoques y planteamientos teóricos y metodológicos; de igual modo, se abordan distintos contenidos (cálculo, geometría, ordenadores, etc.) en diferentes niveles educativos.

Algunos trabajos plantean problemáticas de índole general sobre la construcción del conocimiento matemático y su enseñanza. Es el caso del primer artículo, firmado por mí, en el que se plantea por un lado la insuficiencia de los enfoques piagetiano y cognitivo, centrados en el sujeto y en la universalidad del razonamiento formal, para dar respuesta a los problemas que plantea tanto el aprendizaje como la enseñanza de los conceptos matemáticos. Un planteamiento alternativo se basa en incorporar los planteamientos de la psicología cultural. Desde esta perspectiva, el concocimiento se originaría en contextos de actividad práctica, social y culturalmente organizadas. En el artículo se defiende la idea de que el razonamiento matemático y lógico-deductivo no es sino una forma específica, entre otras, de discurso; por lo tanto, ni «la mejor» ni «la más racional». Para enseñar esa forma de discurso de forma significativa, es necesario que el maestro tenga en cuenta otras formas de discurso, menos formalizadas y más vinculadas a la actividad cotidiana, que posee el alumno.

Abundando en este enfoque de la construcción cultural del conocimiento matemático, Pablo del Río plantea en su artículo un aspecto que nos parece especialmente relevante, como es la función de los materiales, entendidos como mediadores o instrumentos psicológicos en el sentido vygostkiano. Dichos instrumentos o materiales son «mediadores» porque permiten una primera representación mental, aunque ésta sea muy cercana a lo físico o técnico. Diversos tipos de materiales cumplirían una función de acercamiento a la realidad a través de una arquitectura más simple que facilitaría al alumno pasar de los hechos y las cosas a los símbolos que se refieren a esas cosas. En el caso concreto de las matemáticas este aspecto es especialmente importante, porque la utilización de recursos enactivos y códigos naturales facilitaría la posterior traducción a códigos notacionales más formales y abstractos sin perder el significado. Para ilustrar dicho enfoque, Pablo del Río propone algunos ejemplos de cómo dichos materiales pueden ser utilizados en la enseñanza de contenidos geométricos.

En todo proceso de instrucción formal se produce una interacción entre el maestro, el alumno y el objeto de conocimiento. La perspectiva de que todo conocimiento se genera en contextos de interacción social, no nos puede hacer olvidar la necesidad de conocer detalladamente los procesos cognitivos que implica el conocimiento del contenido específico que se enseña, del objeto de conocimiento.

Los artículos de E. Bedoya y M. Orozco, y de J. Deulofeu se orientan hacia la descripción de las estrategias y procedimientos que utilizan los niños en la 
adquisición de dos contenidos matemáticos especialmente relevantes: el del sistema de numeración posicional en el caso de Bedoya y Orozco y el del aprendizaje del lenguaje gráfico para representar funciones en el de Deulofeu.

En el trabajo de E. Martí se presenta una reflexión sugerente sobre un tema controvertido: el de la utilidad del ordenador para aprender matemáticas. Relativizando el posible impacto, muchas veces sobrevalorado, que el uso del ordenador puede tener para dicho aprendizaje, Martí sugiere, ilustrándolo con ejemplos, cuatro aspectos para los que el uso del ordenador podría ser relevante: fomentar la interactividad y la comunicación, integrar diferentes medios simbólicos, poner en relación el conocimiento declarativo y procedimental del alumno y ayudar a la resolución de problemas.

$\mathrm{El}$ artículo de $\mathrm{E}$. Bassedas nos sumerge de lleno en la práctica del aula, describiendo una experiencia de aprendizaje del cálculo en los niveles de educación infantil y primer ciclo de primaria, basada utilización de juegos matemáticos que se trabajan en pequeños grupos o talleres. El interés del trabajo reside sobre todo en la descripción de la metodología de trabajo seguida en el aula. La elaboración de pautas de observación permite al maestro un seguimiento y una evaluación continua de los procedimientos y estrategias usadas por los alumnos, que le ayuda a la vez retroalimentar su propia forma de intervención.

Finalmente, hemos incorporado en este número dos traducciones del informe coordinado por $\mathrm{G}$. Howson y B. Wilson sobre la enseñanza de las matemáticas en la década de los noventa.

En el primero de ellos se aborda el problema del impacto que los cambios sociales y tecnológicos ejercen sobre la escuela, los retos que ello supondrá en el aula y la presión que todo ello ejercerá sobre el maestro, a quien se exigirá el desempeño de nuevos roles para cumplir con dichas expectativas.

En el segundo, y último de los artículos de este volumen, se aborda el problema de los contenidos matemáticos que deben ser enseñados, así como las diferentes alternativas para su tratamiento en el currículum. Probabilidad, Estadística, Geometría, Aplicaciones, Calculadoras y Ordenadores son algunos de los contenidos abordados.

Esperamos que todos estos trabajos contribuyan a clarificar cuestiones y abriré preguntas que permitan seguir avanzando e innovando tanto a nivel teórico como en el trabajo en las aulas. 